\title{
Status of the European Extremely Large Telescope (E-ELT)
}

\author{
Guy J. Monnet
}

European Southern Observatory, Karl-Schwarzschild-Straße 2, D-85748 Garching, Germany email: gmonnet@eso.org

\begin{abstract}
The European initiative for an Extremely Large Telescope is presented. During the past year the transition from the $100 \mathrm{~m}$ OWL concept to a $30 \mathrm{~m}$ to $42 \mathrm{~m}$ diameter telescope has been made. The history and the current status of this development is described.
\end{abstract}

Keywords. telescopes

\section{The prehistory}

Conceptual studies for a new generation of Extremely Large Telescopes, or 'ELT' in short, started quite early in Europe. A university consortium led by Lund University produced the EURO-50 concept in the 1998 to 2003 period, while ESO conducted the OWL conceptual development from 1997 to 2005. At 50 m diameter, EURO-50 had about the absolute maximal size that a 'classical' telescope, based on a segmented aspheric primary, could attain, while OWL at a $60 \mathrm{~m}$ to $100 \mathrm{~m}$ size with segmented spherical primary and secondary mirrors has explored the absolute maximal possible size allowed by mass production. Both have been conceived as general-use facilities, combining large flux collection and exquisite image quality down to the diffraction limit and, consequently, incorporate intrinsic Adaptive Optics correction capabilities.

A European-wide technological programme regrouping close to ninety research institutes and industrial companies has been established to develop and validate the many enabling technologies that are needed to produce such a challenging facility with its very large collecting area and advanced instrumentation. It combines basic R\&D conducted under the aegis of OPTICON (<http://www.astro-opticon.org/>), the FP6 EC-funded Optical Infrared Coordination Network for Astronomy, and the FP6 EC-funded ELT Design Study (<http://www.eso.org/projects/elt-ds/>) for the validation of the many crucial subsystems of any ELT.

\section{The E-ELT scientific case}

In parallel and with close coordination with the conceptual studies, more than one hundred astronomers have built a scientific case for Extremely Large Telescopes under the aegis of OPTICON (<http://www-astro.physics.ox.ac.uk/ imh/ELT/>). Synergy with future ground and space based capabilities, like ALMA and JWST, was taken into account. Main ELT scientific challenges are found in the areas of planetary systems detection and characterisation, study of resolved stellar populations up to the Virgo cluster, study of high-redshift galaxies and detection of the first lights, and use of astronomical objects to probe the frontiers of physics (black holes, quantum gravity effects ... ). The science case is continuously being refined and its latest version, geared towards the European ELT, can be found at <http://www.eso.org/projects/e-elt/publications.html>. 


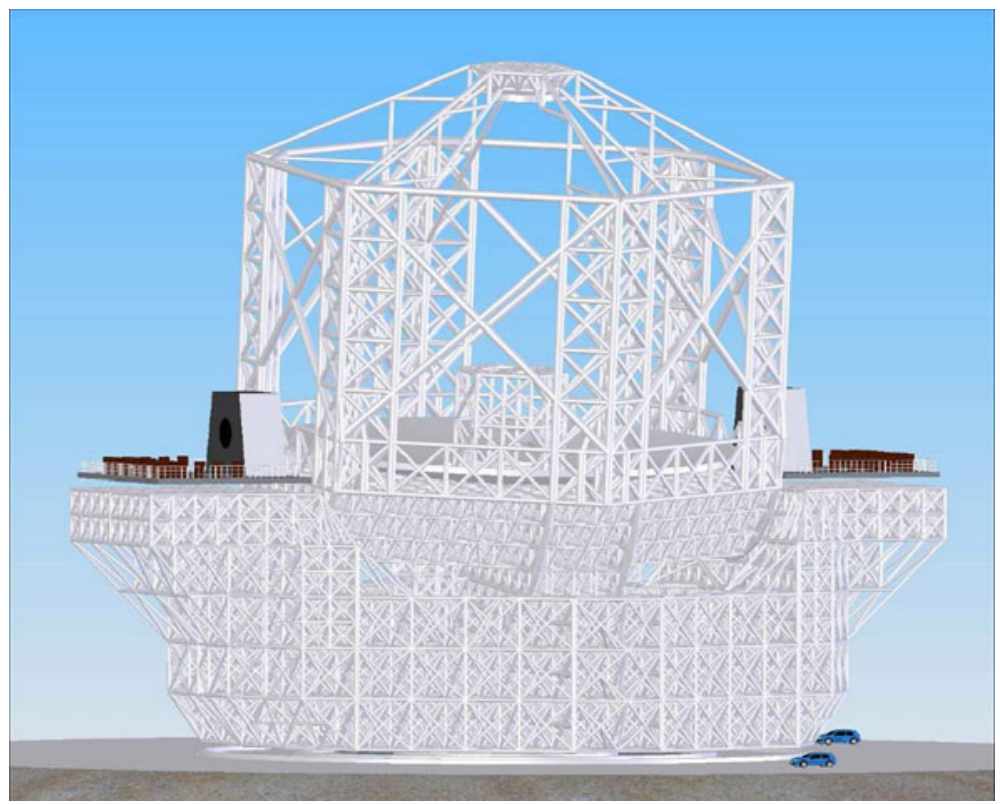

Figure 1. Possible mechanical design for an E-ELT

\section{The E-ELT genesis}

As mandated by its Council in December 2004, ESO has embarked into the Basic Reference Design (BRD) for the European ELT (<http://www.eso.org/projects/e-elt/>). The effort is fully coordinated with the continuing science definition (OPTICON) and technological development (OPTICON \& ELT Design Study). It is conducted with the help of working groups with extensive help from community astronomers. The goal is to get a high-performance science-optimised general-use facility for the European community. The telescope size is envisioned to be in the $30 \mathrm{~m}$ to $42 \mathrm{~m}$ range and will be set by the cost/risk versus performance ratio. The BRD will be presented to the community at large in Marseilles at the end of November (<http://www.elt2006.org>) and early December to the ESO Council for decision on launching the Project.

\section{Conclusion}

Within this ambitious European programme, there is ample need for cooperation with the equally ambitious developments occurring on the other side of the Atlantic. E-ELT, GMT and TMT share most of the same enabling technology needs and coordination in this area shall be maintained and ideally much augmented. There is also potential for useful complementarities between these future facilities and optimising their combined science output does look like a promising approach, which deserves to be seriously explored. 